\title{
Study of Various Factors for Enhancement of Artemisinin in Artemisia Annua Hairy Roots
}

\author{
Nivedita Patra, A. K. Srivastava, and Shilpi Sharma
}

\begin{abstract}
Artemisinin is an alternate anti-malarial drug which is widely used in the cure of multi-drug resistant Plasmodium falciparum malaria. In this study, enhancement of artemisinin content by using several elicitors and precursors was attempted initially by using one at a time (OVAT) approach. The most potent elicitor(s) \& precursor were thereafter identified by the detailed analysis of the responses with respect to biomass and bioactive compound with respect to the concentrations of different effectors. Central Composite Design (CCD) was thereafter used to identify the antagonistic or synergistic effects of high and low concentrations of different precursors and elicitors. The maximum artemisinin content obtained was $3.45 \mathrm{mg} / \mathrm{g}$ on $15 \mathrm{~d}$ by using the elicitor methyl jasmonate $(40 \mu \mathrm{g} / \mathrm{l})$ and the precursors casein acid hydrolysate $(50 \mu \mathrm{g} / \mathrm{l})$, sodium acetate $(500 \mu \mathrm{g} / \mathrm{l})$. Effect of addition time of elicitors and precursors on overall optimum biomass growth and artemisinin yield and productivity was also established.
\end{abstract}

Index Terms-Artemisinin, hairy root culture, elicitors, precursors, OVAT, central composite design.

\section{INTRODUCTION}

Artemisinin is a pharmaceutical compound of immense therapeutic importance. It is commonly used to treat multidrug resistant Plasmodium falciparum Malaria (Cerebral malaria). Presently the commercial production of this compound is through natural plant Artemisia annua. Therefore the supply of this drug is much lower than its demand particularly because of its low content in plants and also the production is subject to seasonal and commercial limitations. Chemical synthesis of Artemisinin is highly complex and uneconomical. Alternate production protocols are sought to supplement Artemisnin production.

Plant cell /hairy root cultivation have been reported to be the ideal production technologies and are being researched extensively. Hairy roots in particular are more stable artificial roots \& are obtained by genetic transformation of different plant parts of Artemisia annua using Agrobacterium rhizogenes [1]. Literature reports indicate that content of artemisinin in hairy roots can be upto $0.4 \%$ g/g DW [2]. Mass scale propagation of hairy roots in shake flasks and various bioreactor cultivations have the capability to mass produce the biomass in shorter time period leading to higher artemisinin content \& significantly high productivity [3].

Manuscript received March 8, 2013; revised May 10, 2013.

Nivedita Patra, A. K. Srivastava, and Shilpi Sharma are with Department of Biochemical Engineering \& Biotechnology, Indian Institute of Technology, Hauz Khas, New Delhi -110016, India (e-mail: nivedita.nitdgp@gmail.com)
There has been a growing demand to optimize artemisinin content by the growing hairy root mass. Several strategies have been adopted in the literature for enhancement of artemisinin content from cell and hairy root cultivation. Reports have suggested significant rise in artemisinin content by manipulation of Nitrogen (in form of Urea) added to fields per hectare which led to the maximum artemisinin production of $27.5 \mathrm{mg} / 100 \mathrm{gm}$ dried leaves. It has been shown that addition of some inhibitors in liquid medium led to inhibition of Artemisinin formation in plantlets e.g., the addition of IPP inhibitors mevilonin (mevalonate pathway inhibitor) and Fosmidomycin (nonmevalonate pathway inhibitor) significantly reduced Artemisinin content [4]. Enhancement of Artemisinin by addition of sterol biosynthesis inhibitor, miconazole has been reported in the literature. DMSO in the liquid medium has been indicated to enhance Artemisinin in plantlets by 6 times as compared to controls. It enhanced phage transcription and has been reported to alter cellular functions like $\mathrm{Zn}$ uptake, respiration, RNA and protein metabolism. Shoot cultures have been initiated from leaves using MS medium with 3\% sucrose, $0.5 \mathrm{mg}$ 6-BA, $0.05 \mathrm{mg}$ $\mathrm{NAA}$ and $8 \mathrm{~g}$ agar per liter. In shoot culture artemisinin content increased with increasing sucrose concentration from 0-30 g/l which declined thereafter. High content of Artemisinin in hairy root cultures of A. annua featured evidence of production of Artemisinin outside trichomes. Trichomes have been known as the sites for synthesized artemisinin in leaves and flowers.

\section{PROCEDURE}

\section{A. Plant Material and Induction of Hairy Roots}

Seeds and plantlets of Artemisia апnиa were obtained from CIMAP (Lucknow). Induction of hairy roots was done according to the protocol reported in literature [5]. Apical meristem explants of Artemisia annua were surface sterilized in $10 \%$ sodium hypochlorite for 5 minutes. After being washed three times with sterilized water, the explants were immersed in $70 \%$ ethanol for 1 minute. The explants were incubated in hormone-free MS medium containing $3 \%$ sucrose, at 25 degree Celsius overnight under white light. After overnight incubation in MS media the explants were infected with Agrobacterium rhizogenes strain LBA 301 and cultured on MS medium at $25^{\circ} \mathrm{C}$ for 2 days in dark and then transferred to MS medium with antibiotic (Cefotaxime, $500 \mathrm{mg} / \mathrm{l}$ ). Infection was done by dipping the cut ends of apical meristem explants in saturated Agrobacterium cultures for 20 minutes. The bacteria free hairy roots were subcultured in MS media every three 
weeks.

\section{B. Confirmation of Transformed Status Using PCR}

Molecular characterisation of hairy root was done using PCR for rol A gene (root locus gene).The confirmation of transformed status of plant tissue was done by a method standardised earlier for A. indica hairy roots [6]. In brief, genomic DNA was isolated from hairy roots. This was used as template for specific PCR reaction with specific primers for amplification of rol A. PCR with genomic DNA from non-transformed plant roots was used for negative control experiment and PCR with plasmid DNA of Agrobacterium rhizogenes was used as positive control experiment.

\section{Effect of Elicitors and Precursors}

The effect of following elicitors and precursors were studied on the growth and artemisinin content of the hairy roots The selection of elicitors and precursors and their range of concentrations was done by extensive literature survey.

- $\quad$ Sodium acetate $-10,25,50 \mathrm{mg} / \mathrm{l}$

- Mevalonic acid lactone - 10, 20,50 mg/l

- Cholesterol - 10, 20,30 mg/1

- Casein acid hydrolysate - 0.1, 0.5, $1.0 \mathrm{~g} / \mathrm{l}$

- GA3 - 1-10 ppm

- Methyl jasmonate - 10, 20, $30 \mathrm{mg} / \mathrm{l}$

- $\quad$ Salicylic acid - 10, 30, $50 \mathrm{mg} / \mathrm{l}$

- Calcium chloride - 50, $100 \mathrm{mg} / \mathrm{l}$

- $\quad$ Yeast extract - 10, 20,50 mg/l

- Chitosan - 10, 20, $50 \mathrm{mg} / \mathrm{l}$

\section{Statistical Optimisation of Combined Addition of} Elicitor and Precursor

After the identification of the most potent elicitors and precursor, Statistical optimization tools (Plackett Burman Method followed by Response Surface Analysis) was used to establish the optimum concentrations of the selected elicitors and precursors. These were then used as a combined strategy for enhancing the artemisinin concentration. A $2^{4}$ full factorial Central Composite Design (CCD) for independent variables was employed for optimization. A second order polynomial model was developed based on studies of the responses of different effectors.

\section{RESULTS}

\section{A. Development and Maintenance of Hairy Root Culture of Artemisia Annua}

Elite seeds, particularly of high yielding variety of Artemisia annua were procured from CIMAP Lucknow, India. Hairy roots were induced from the in vitro plantlets using Agrobacterium rhizogenes mediated transformation. Selection of a suitable root line was based on the comparison of growth index (defined as Growth Index (G.I) $=($ Final fresh weight- Initial fresh weight $) /$ Initial fresh weight) and Artemisinin content (mg/g dry weight). The selected root line was maintained by sub-culturing every 2 weeks in MS medium as this medium was reported to minimise polyphenol production and therefore less subculture cycles were required with this medium [7]. A high artemisinin containing hairy root line $(1.8 \mathrm{mg} / \mathrm{g}$ dry cell weight basis) named CIM 22, derived from in vitro plants of Artemisia annua was eventually selected for further studies.

\section{B. PCR Study for Confirmation of Transformed Status of Rootline Selected}

The selected Hairy root line indicated the presence of rolA gene on the template DNA after PCR cycle, which was visible as an amplified band (300 bp) during the agarose gel electrophoresis of the PCR analysis. The size of the amplified product was confirmed by using a positive control at the same PCR conditions (plasmid DNA from Agrobacterium rhizogenes LBA 301). This led to the establishment of the transformed nature of the hairy root line CIM 22.

\section{Effect of Elicitors and Precursors}

The production of secondary metabolites can be increased by the addition of its biosynthetic precursors in the culture medium. The effect of precursors like casein acid hydrolysate, sodium acetate and mevalonic acid lactone were studied. The precursor effect on artemisinin content has been summarised in Fig. 1. It was observed that casein acid hydrolysate and sodium acetate had positive significant effect on artemisinin content in the growing hairy roots. It has been reported in the literature that sodium acetate in the medium causes damage to plant cells and thus cause the release of oligogalacturonides. This signals tissue damage and causes the enhancement of defence related molecules (Phytoalexins) in the cell. Thus sodium acetate acts as an elicitor than a precursor. Casein acid hydrolysate, on the contrary, is a complex nitrogenous source with vitamins and minerals and therefore enhances growth and artemisinin content.

The effect of different concentrations of various elicitors like calcium chloride, methyl jasmonate, salicylic acid and yeast extract were seen on artemisinin content in hairy roots. The result of the study is summarized in Fig. 2. Methyl jasmonate turned out to be the most potent elicitor for enhancement of artemisinin content. Methyl jasmonate has been reported to act as a quick messenger to neighboring undamaged plant cells, thus prompting them to produce defensive chemicals before they are attacked.

\section{Effect of precursor addition on Artemisinin production by $A$. annua hairy root cultures}

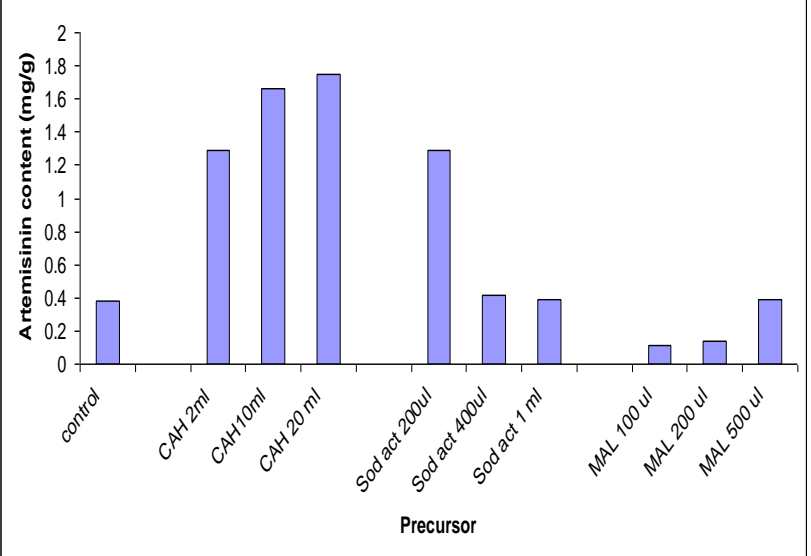

Fig. 1. Effect of precursors on artemisinin production 
D. Statistical Optimization of the Selected Elicitor and Precursor in the Optimized Culture Medium

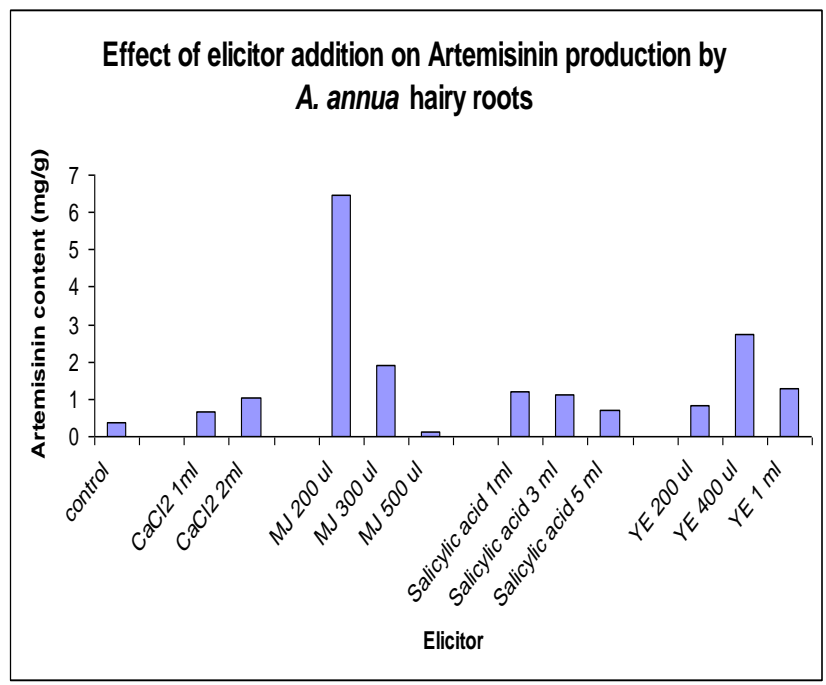

Fig. 2. Effect of elicitors on artemisinin production

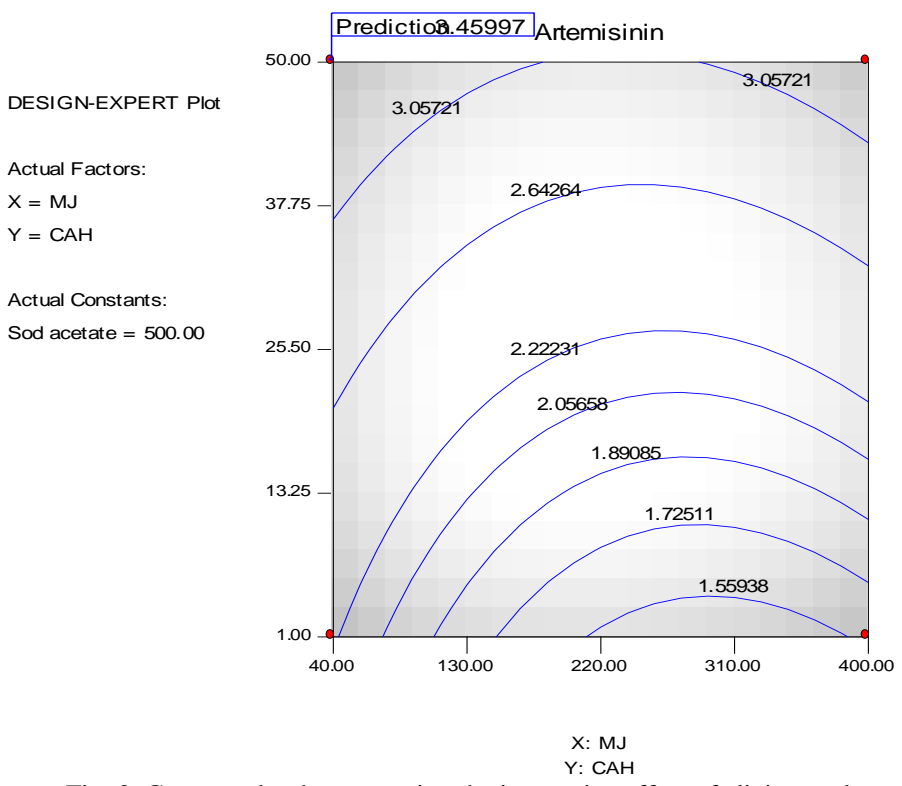

Fig. 3. Contour plot demonstrating the interactive effect of elicitor and precursor on artemisinin production in a. annua hairy roots

\section{E. Optimised Elicitor Concentration for Maximum Artemisinin}

The interaction between low and high concentrations of casein acid hydrolysate, sodium acetate and methyl jasmonate were studied in detail using the statistically designed experimental recipe of RSM (Table I). Twenty experiments were designed using above mentioned three effectors and the results of responses were studied with respect to biomass and Artemisinin content. The above experiments formed the basis for parameter evaluation of the models for artemisinin (Equation 1) \& Biomass (Equation 2). These model equations were solved for different CONCENTRATIONS OF EFFECTORS methyl jasmonate \& casein acid hydrolysate keeping sodium acetate constant. The contour plots emerging out of above analysis are described in the Figure 3 (Artemisnin). From the analysis of above model simulations and contour plots, the optimal concentration of the ELICITORS AND PRECURSORS emerged as indicated below:

Methyl jasmonate: $40 \mu \mathrm{g} / \mathrm{L}$
Casein acid hydrolysate: $50 \mu \mathrm{g} / \mathrm{L}$

Sodium acetate: $500 \mu \mathrm{g} / \mathrm{L}$

Model for response:

$$
\begin{aligned}
& \text { Artemisnin }=+1.60-0.27 \times \mathrm{A}+0.18 \times \mathrm{B}+0.23 \times \mathrm{C}+ \\
& 0.38 \times \mathrm{A}^{2}+0.088 \times \mathrm{B}^{2}+0.37 \times \mathrm{C}^{2}+0.13 \times \mathrm{A} \times \mathrm{B}+ \\
& 0.077 \times \mathrm{A} \times \mathrm{C}+0.57 \times \mathrm{B} \times \mathrm{C} \\
& \text { Biomass }=+0.69-0.22 \times \mathrm{A}-0.17 \times \mathrm{B}+9.227 \mathrm{E}-03 \times \mathrm{C}+ \\
& 0.14 \times \mathrm{A}^{2}+0.077 \times \mathrm{B}^{2}+0.040 \times \mathrm{C}^{2}+0.10 \times \mathrm{A} \times \mathrm{B}+ \\
& 0.022 \times \mathrm{A} \times \mathrm{C}-0.06 \times \mathrm{B} \times \mathrm{C}
\end{aligned}
$$

\begin{tabular}{|c|c|c|c|c|c|}
\hline S. No. & $\begin{array}{l}\text { MJ } \\
(\mathrm{ug} / \mathrm{ml})\end{array}$ & $\begin{array}{l}\text { CAH } \\
(\mathrm{ug} / \mathrm{ml})\end{array}$ & $\begin{array}{l}\text { Sod. } \\
\text { Acetate } \\
(\mathrm{ug} / \mathrm{ml})\end{array}$ & $\begin{array}{l}\text { Biomass } \\
(\mathrm{g} / \mathrm{l})\end{array}$ & $\begin{array}{l}\text { Arte } \\
(\mathrm{mg} / \mathrm{g})\end{array}$ \\
\hline 1. & 40 & 50 & 50 & 0.95 & 1.8 \\
\hline 2. & 220 & 25.50 & 275 & 0.7 & 1.3 \\
\hline 3. & 400 & 1.00 & 50 & 0.69 & 1.58 \\
\hline 4. & 220 & 25.50 & 275 & 0.7 & 1.3 \\
\hline 5. & 400 & 1.00 & 500 & 0.95 & 2.1 \\
\hline 6. & 40 & 50 & 500 & 0.87 & 4.28 \\
\hline 7. & 400 & 50 & 500 & 0.64 & 4.91 \\
\hline 8. & 220 & 25.50 & 275 & 0.70 & 1.3 \\
\hline 9. & 40 & 1 & 50 & 1.43 & 1.80 \\
\hline 10. & 400 & 50 & 50 & 0.75 & 1.13 \\
\hline 11. & 220 & 25.50 & 275 & 0.7 & 1.3 \\
\hline 12. & 40 & 1 & 500 & 1.48 & 3 \\
\hline 13. & 220 & 66.70 & 275 & 0.61 & 1.38 \\
\hline 14. & 0 & 25.50 & 275 & 1.16 & 2.63 \\
\hline 15. & 220 & 25.50 & 0 & 0.74 & 4.45 \\
\hline 16. & 522.72 & 25.50 & 275 & 0.69 & 1.72 \\
\hline 17. & 220 & 25.50 & 275 & 0.7 & 1.3 \\
\hline 18. & 220 & 25.50 & 275 & 0.7 & 1.3 \\
\hline 19. & 220 & 0 & 275 & 0.92 & 2.42 \\
\hline 20. & 220 & 25.50 & 653.40 & 0.79 & 1.25 \\
\hline
\end{tabular}

where $\mathrm{A}=$ Methyl Jasmonate, $\mathrm{B}=$ Casein acid hydrolysate, $\mathrm{C}=$ Sodium acetate

TABLE I: EXPERIMENTAL RECIPE IN CENTRAL COMPOSITE DESIGN (CCD) FOR ELICITOR OPTIMIZATION

\section{CONCLUSION}

The maximum artemisinin content obtained was 3.45 $\mathrm{mg} / \mathrm{g}$ on $15^{\text {th }}$ day of cultivation in shake flask by using the elicitor methyl jasmonate $(40 \mu \mathrm{g} / \mathrm{l}) \&$ precursors casein acid hydrolysate $(50 \mu \mathrm{g} / \mathrm{l})$, sodium acetate $(500 \mu \mathrm{g} / \mathrm{l})$ as compared to $0.4 \mathrm{mg} / \mathrm{g}$ artemisinin in control experiment of hairy root cultivation with no addition of elicitors.

It was also established that maximum artemisinin production was observed when elicitation \& precursor addition was done on $12^{\text {th }}$ day of cultivation of hairy roots $\&$ 
the growth was continued till 92 hours thereafter.

\section{REFERENCES}

[1] S. Srivastava and A. K. Srivastava, "Hairy root culture for massproduction of high-value secondary metabolites," Critical Reviews in Biotechnology, vol. 27, pp. 1-15, Jan. 2007.

[2] P. J. Weathers, R. D. Cheetham, E. Follansbee, and T. Teoh, "Artemisinin production by transformed roots of Artemisia аппиа," Biotechnology Letters, vol. 16, pp. 1281-1286, Dec. 1994.

[3] C. Z. Liu, Y. C. Wang, F. Ouyang, H. C. Ye, and G. E. Li, "Production of artemisinin by hairy root cultures of Artemisia annua L.," Biotechnology Letters, vol. 19, pp. 927-930, September 1997.

[4] M. Towler and Pamela Weathers, "Evidence of artemisinin production from IPP stemming from both the mevalonate and the nonmevalonate pathways," Journal of Plant Cell Reports, vol. 26, pp. 2129-2136, Dec. 2007.

[5] W. Putalun, W. Luealon, W. De-Eknamkul, H. Tanaka, and Y. Shoyama, "Improvement of artemisinin production by chitosan in hairy root cultures of Artemisia аппиа L.," Biotechnology Letters, vol 29, pp. 1143-1146, July 2007.

[6] S. Srivastava and A. K. Srivastava, "In vitro cultivation of Azadirachta indica in nutrient mist bioreactor," Applied Biochemistry and Biotechnology, vol. 166, pp. 365-378, Jan. 2012.

[7] T. Murashige and F. Skoog, "A revised medium for rapid growth and bioassays with tobacco tissue cultures," Physiology Plant, vol. 15, pp. 473-497, July 1962.

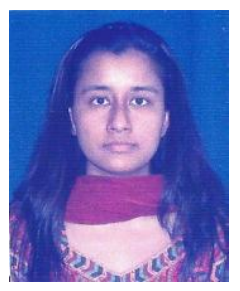

Nivedita Patra had done M.Tech in Biotechnology from School of Biotechnology, GGSIPU, New Delhi. Ms. Patra has also worked as a lecturer in the Biotechnology Department, National Institute of Technology Durgapur from 2007-2008.

Currently she is pursuing her Ph.D. degree at the Department of Biochemical Engineering and Biotechnology IIT Delhi on a topic entitled "Development and mass scale propagation of Artemisia annua hairy root culture in a suitable bioreactor system for Artemisinin production" since the past 5 years. She is about to submit her Ph.D. thesis. She has presented several papers at national and international conferences and published 1 book chapter entitled "Statistical media optimization for enhanced biomass and Artemisinin production in Artemisia аппиа hairy roots" in a book entitled Chemistry of Phytopotentials: Health Energy and Environment Perspectives (Springer, Germany). She has also won best poster prizes at International conference (CPHEE-2011 Agra) and IIT Delhi open house $\mathrm{I}^{2} \mathrm{Tech}-2012$.

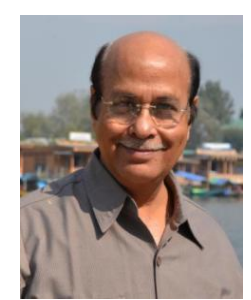

Ashok Kumar Srivastava received Ph.D. degree from the McGill University, Montreal (Quebec) in $1990 \mathrm{He}$ has extensive Industrial - Research and Teaching experience in the area of Biochemical Engineering and Biotechnology. Dr. Srivastava is Professor in IIT Delhi and has been working in IIT Delhi since 1991.

$\mathrm{He}$ has received several awards and scholarships, important amongst them is National Overseas Scholarship award of MHRD, New Delhi for Ph.D. studies (1985-1990) \& UNESCO fellowship award (6 months) for advanced studies in Biochemical Engineering at Biochemical Engineering Group, Delft University, The Netherlands.

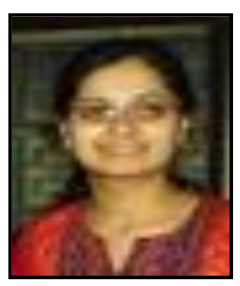

Shilpi Sharma is working as an assistant professor in the Department of Biochemical Engineering and Biotechnology, IIT Delhi, New Delhi Her main area of research is Functional Microbial Ecology in terrestrial and waste water treatment systems 